\title{
Dynamic behavior of traveling wave solutions for new couplings of the Burgers equations with time-dependent variable coefficients
}

Husein M Jaradat ${ }^{*}$

"Correspondence:

husseinjaradat@yahoo.com

Department of Mathematics, Al

al-Bayt University, Mafraq, Jordan Department of Mathematics and

Applied Sciences, Dhofar University,

Salalah, Oman

\section{Springer}

\begin{abstract}
In this paper, we develop the nonlinear integrable couplings of Burgers equations with time-dependent variable coefficients. A new simplified bilinear method is used to obtain new multiple-kink solutions and multiple-singular-kink solutions for this system. The proposed system is a generalization model in ocean dynamics, plasma physics and nonlinear lattice. The effects of time-variable coefficients on the velocity, phase and amplitude are given. The solitonic propagation and collision are discussed by the graphical analysis and characteristic-line method.
\end{abstract}

Keywords: Hirota bilinear method; multiple-kink solutions; coupled Burgers equations

\section{Introduction}

The classical coupled Burgers equations (CBE) [1-3] with time $t$ and space $x$ derivatives are given by

$$
\left.\begin{array}{l}
v_{t}-v_{x x}-2 v v_{x}+a(v w)_{x}=0, \\
w_{t}-w_{x x}-2 w w_{x}+b(v w)_{x}=0
\end{array}\right\}
$$

where $t>0, x$ is a horizontal coordinate space and $a, b$ are constants. The coupled Burgers equations $(\mathrm{CBE})$ arise in a large number of applications in physics, engineering and mathematical problems. Some if these applications are plasma physics, fluid mechanics, optic, solid state physics, chemical physics, etc. [1-3]. Many researchers in applied mathematics give great attention to finding the analytical, approximation and exact solutions of $\mathrm{CBE}$ by different methods such as variational iteration method [4], Adomian-Pade technique [5], differential transformation method [2], exponential function method in rational form [6], homotopy analysis method [7], modified extended direct algebraic (MEDA) method [8], first integral method [9], reduced differential transform method [10] and the Hirota bilinear method [11].

(c) The Author(s) 2017. This article is distributed under the terms of the Creative Commons Attribution 4.0 International License (http://creativecommons.org/licenses/by/4.0/), which permits unrestricted use, distribution, and reproduction in any medium, provided you give appropriate credit to the original author(s) and the source, provide a link to the Creative Commons license, and indicate if changes were made. 
In this paper, we develop the classical coupled Burgers equations (1) to derive nonlinear $\mathrm{n}$-coupled Burgers equations with time-variable coefficients (nc-BE) in the form

$$
\left.\begin{array}{l}
\left(w_{1}\right)_{t}-\alpha_{1}(t)\left(w_{1}\right)_{x x}-\beta_{1}(t) w_{1}\left(w_{1}\right)_{x}+\gamma_{1}(t)\left(w_{1} w_{2} w_{3} \cdots w_{n}\right)_{x}=0, \\
\left(w_{2}\right)_{t}-\alpha_{2}(t)\left(w_{2}\right)_{x x}-\beta_{2}(t) w_{2}\left(w_{2}\right)_{x}+\gamma_{2}(t)\left(w_{1} w_{2} w_{3} \cdots w_{n}\right)_{x}=0 \\
\left(w_{3}\right)_{t}-\alpha_{3}(t)\left(w_{3}\right)_{x x}-\beta_{3}(t) w_{3}\left(w_{3}\right)_{x}+\gamma_{3}(t)\left(w_{1} w_{2} w_{3} \cdots w_{n}\right)_{x}=0 \\
\vdots \\
\left(w_{n}\right)_{t}-\alpha_{n}(t)\left(w_{n}\right)_{x x}-\beta_{n}(t) w_{n}\left(w_{n}\right)_{x}+\gamma_{n}(t)\left(w_{1} w_{2} w_{3} \cdots w_{n}\right)_{x}=0 .
\end{array}\right\}
$$

When $n=2, \alpha_{1}(t)=\alpha_{2}(t)=1, \beta_{1}(t)=\beta_{2}(t)=2, \gamma_{1}(t)=a$, and $\gamma_{2}(t)=b$, the coupling (2) reduces to the classical coupled (1). The objectives of this work are the following:

1. Derive a form of nonlinear n-coupled Burgers equations (2).

2. Show that it has multiple-kink solutions and multiple-singular-kink solutions by using the Backlund transformations and simplified Hirota's method [12-26].

In this study, we need the following conditions on (2):

$$
\alpha_{j}(t)=b_{j} \beta_{j}(t), \quad j=1,2,3, \ldots, n,
$$

where $b_{j}$ are arbitrary constants.

Finally, we define the 'kink' as a type of solitons which is in the form tanh, not $\tanh ^{2}$. In a kink, we take the limit when $x$ approaches infinity. The answer is a constant, unlike solitons where the limit goes to 0 . Solitons are solutions in the form of sech and sech ${ }^{2}$. The graph of the soliton is a wave which is positive. It is unlike the periodic solutions sine, cosine, etc. In trigonometric functions, waves go above and below the horizontal line [27].

This paper is organized as follows. A new N-kink solutions and N-singular-kink solutions for the nc-BE system (2) are constructed in Sections 1 and 2. The effect of the variable coefficients and the collision behavior and propagation properties are discussed in Section 3. Finally, conclusions are given in Section 4.

\section{Multiple-kink solutions for the nc-BE system}

In this section, we use the simplified bilinear method [28-30] to construct multiple-kink solutions of nc-BE system (2). If we substitute

$$
\begin{aligned}
& w_{j}(x, t)=e^{\phi_{i j}(x, t)}, \quad j=1,2,3, \ldots, n, \\
& \phi_{i j}(x, t)=s_{i} x-\delta_{i j}(t)
\end{aligned}
$$

into the linear terms of Eq. (2), we get the dispersion relation as follows:

$$
\delta_{i j}(t)=-\int s_{i}^{2} \alpha_{j}(t) d t
$$

Thus,

$$
\phi_{i j}(x, t)=s_{i} x+\int s_{i}^{2} \alpha_{j}(t) d t
$$

Assume that the multiple-kink solutions of (2) are

$$
w_{j}(x, t)=C_{j}\left(\ln a_{j}(x, t)\right)_{x}, \quad j=1,2,3, \ldots, n .
$$


For single-kink solutions, the $a_{j}(X, T)$ is given by

$$
a_{j}(x, t)=1+e^{\phi_{1 j}(x, t)}=1+e^{s_{1} x+\int s_{1}^{2} \alpha_{j}(t) d t} .
$$

Substitute Eqs (6) and (7) into Eq. (2), then solving for $C_{1}, C_{2}, C_{3}, \ldots, C_{n}$, the non-zero solution is given by

$$
C_{j}=\frac{2 \alpha_{j}(t)}{\beta_{j}(t)}, \quad j=1,2,3, \ldots, n
$$

To obtain a numerical value of $R_{j}$, we set the constraints $\frac{\alpha_{j}(t)}{\beta_{j}(t)}=b_{j}, j=1,2,3, \ldots, n$, where $b_{j}$ are arbitrary constants. Now, substitute Eq. (8) into Eq. (6), to obtain the single-kink solutions for (2) as follows:

$$
\left.\begin{array}{rl}
w_{j}(x, t) & =2 s_{1} \frac{\alpha_{j}(t)}{\beta_{j}(t)} \times \frac{e^{\phi_{1 j}(x, t)}}{\left(1+e^{\phi_{1 j}(x, t)}\right)} \\
& =2 s_{1} \frac{\alpha_{j}(t)}{\beta_{j}(t)} \times \frac{e^{s_{1} x+\int s_{1}^{2} \alpha_{j}(t) d t}}{1+e^{s_{1} x+\int s_{1}^{2} \alpha_{j}(t) d t}} \\
& =s_{1} \frac{\alpha_{j}(t)}{\beta_{j}(t)}\left[1+\tanh \frac{\phi_{1 j}(x, t)}{2}\right], \quad j=1,2,3, \ldots, n,
\end{array}\right\}
$$

where

$$
\phi_{1 j}(x, t)=s_{1} x+\int s_{1}^{2} \alpha_{j}(t) d t
$$

To obtain the two-kink solutions, let

$$
\left.\begin{array}{rl}
a_{j}(x, t) & =1+e^{\phi_{1 j}(x, t)}+e^{\phi_{2 j}(x, t)}+b_{12} e^{\phi_{1 j}(x, t)+\phi_{2 j}(x, t)} \\
& =1+e^{s_{1} x+\int s_{1}^{2} \alpha_{j}(t) d t}+e^{s_{2} x+\int s_{2}^{2} \alpha_{j}(t) d t}+b_{12} e^{\left(s_{1}+s_{2}\right) x+\left(s_{1}^{2}+s_{2}^{2}\right) \int \alpha_{j}(t) d t}
\end{array}\right\}
$$

where $\phi_{1 j}(x, t)$ and $\phi_{2 j}(x, t)$ are defined in Eq. (5). Using Eqs (10) and (6) and substituting the results in Eq. (2), we obtain the value of phase shift by

$$
b_{12}=0
$$

Hence,

$$
b_{i j}=0, \quad 1 \leq i<j \leq 3 .
$$

Substituting Eqs (11), (10) and (8) into Eq. (6), we obtain two-kink solutions for Eq. (2)

$$
w_{j}(x, t)=2 \frac{\alpha_{j}(t)}{\beta_{j}(t)} \times \frac{s_{1} e^{s_{1} x+\int s_{1}^{2} \alpha_{j}(t) d t}+s_{2} e^{s_{2} x+\int s_{2}^{2} \alpha_{j}(t) d t}}{1+e^{s_{1} x+\int s_{1}^{2} \alpha_{j}(t) d t}+e^{s_{2} x+\int s_{2}^{2} \alpha_{j}(t) d t}}, \quad j=1,2,3, \ldots, n
$$

The three-soliton solutions are determined by

$$
\begin{aligned}
a_{j}(x, t)=1 & +e^{\phi_{1 j}(x, t)}+e^{\phi_{2 j}(x, t)}+e^{\phi_{3 j}(x, t)}+b_{12} e^{\phi_{1 j}(x, t)+\phi_{2 j}(x, t)} \\
& +b_{13} e^{\phi_{1 j}(x, t)+\phi_{3 j}(x, t)}+b_{23} e^{\phi_{2 j}(x, t)+\phi_{3 j}(x, t)}+b_{123} e^{\phi_{1 j}(x, t)+\phi_{2 j}(x, t)+\phi_{3 j}(x, t)},
\end{aligned}
$$


where

$$
b_{i j}=0, \quad 1 \leq i<j \leq 3 .
$$

Proceeding as before, we find

$$
b_{123}=0 \text {. }
$$

Then

$$
a_{j}(x, t)=1+e^{s_{1} x+\int s_{1}^{2} \alpha_{j}(t) d t}+e^{s_{2} x+\int s_{2}^{2} \alpha_{j}(t) d t}+e^{s_{3} x+\int s_{3}^{2} \alpha_{j}(t) d t} .
$$

Thus, the three-kink solution for Eq. (2) is given by

$$
w_{j}(x, t)=2 \frac{\alpha_{j}(t)}{\beta_{j}(t)} \times \frac{s_{1} e^{s_{1} x+\int s_{1}^{2} \alpha_{j}(t) d t}+s_{2} e^{s_{2} x+\int s_{2}^{2} \alpha_{j}(t) d t}+s_{3} e^{s_{3} x+\int s_{3}^{2} \alpha_{j}(t) d t}}{1+e^{s_{1} x+\int s_{1}^{2} \alpha_{j}(t) d t}+e^{s_{2} x+\int s_{2}^{2} \alpha_{j}(t) d t}+e^{s_{3} x+\int s_{3}^{2} \alpha_{j}(t) d t}}, \quad j=1,2,3, \ldots, n .
$$

To this point, we reach the fact that Eq. (2) is completely integrable and $N$-kink solutions exist for $N \geq 1[12,15]$. Moreover, we can obtain $N$-kink solutions as follows:

$$
w_{j}(x, t)=2 \frac{\alpha_{j}(t)}{\beta_{j}(t)} \times \frac{\sum_{i=1}^{N} s_{i} e^{s_{i} x+\int s_{i}^{2} \alpha_{j}(t) d t}}{1+\sum_{i=1}^{N} e^{s_{i} x+\int s_{i}^{2} \alpha_{j}(t) d t}}, \quad j=1,2,3, \ldots, n .
$$

\section{Multiple-singular-kink solutions for the nc-BE system}

In order to obtain the single-singular-kink solutions of Eq. (2), we substitute

$$
w_{j}(x, t)=e^{s_{i} x-\delta_{i j}(t)}, \quad j=1,2,3, \ldots, n,
$$

into the linear part of Eq. (2); as a result, we get

$$
\delta_{i j}(t)=-\int s_{i}^{2} \alpha_{j}(t) d t
$$

Assume that the single-singular-kink solutions of Eq. (2) are

$$
w_{j}(x, t)=C_{j}\left(\ln a_{j}(x, t)\right)_{x}, \quad j=1,2,3, \ldots, n,
$$

where $a_{j}(x, t)$ is given by

$$
a_{j}(x, t)=1-e^{s_{1} x+\int s_{1}^{2} \alpha_{j}(t) d t} .
$$

Substituting Eq. (14) into Eq. (2) and solving for $C_{j}$, we get

$$
\left.C_{j}=2 \frac{\alpha_{j}(t)}{\beta_{j}(t)}, \quad j=1,2,3, \ldots, n .\right\}
$$


Similarly, we set the constraints $\frac{\alpha_{j}(t)}{\beta_{j}(t)}=b_{j}, j=1,2,3, \ldots, n$, where $b_{j}$ are arbitrary constants to obtain a numerical value of $C_{j}$. Then the single-singular-kink solutions of Eq. (2) are

$$
\left.\begin{array}{rl}
w_{j}(x, t) & =-2 s_{1} \frac{\alpha_{j}(t)}{\beta_{j}(t)} \times \frac{e^{\phi_{1 j}(x, t)}}{\left(1-e^{\phi_{1 j}(x, t)}\right)} \\
& =-2 s_{1} \frac{\alpha_{j}(t)}{\beta_{j}(t)} \times \frac{e^{s_{1} x+\int s_{1}^{2} \alpha_{j}(t) d t}}{1-e^{s_{1} x+\int s_{1}^{2} \alpha_{j}(t) d t}} \\
& =s_{1} \frac{\alpha_{j}(t)}{\beta_{j}(t)}\left[1+\operatorname{coth} \frac{\phi_{1 j}(x, t)}{2}\right], \quad j=1,2,3, \ldots, n,
\end{array}\right\}
$$

where

$$
\phi_{1 j}(x, t)=s_{1} x+\int s_{1}^{2} \alpha_{j}(t) d t
$$

The two-singular-kink solutions are obtained by setting

$$
\begin{aligned}
a_{j}(x, t) & =1-e^{\phi_{1 j}(x, t)}-e^{\phi_{2 j}(x, t)}+b_{12} e^{\phi_{1 j}(x, t)+\phi_{2 j}(x, t)} \\
& =1-e^{s_{1} x+\int s_{1}^{2} \alpha_{j}(t) d t}-e^{s_{2} x+\int s_{2}^{2} \alpha_{j}(t) d t}+b_{12} e^{\left(s_{1}+s_{2}\right) x+\left(s_{1}^{2}+s_{2}^{2}\right) \int \alpha_{j}(t) d t} .
\end{aligned}
$$

Substituting Eq. (16) into Eq. (13) and then in Eq. (2), we obtain the phase shift $b_{12}$ as

$$
b_{12}=0
$$

Substitute Eqs (17), (16) and (15) into Eq. (13), then the two-singular-kink solutions for Eq. (2) are

$$
w_{j}(x, t)=-2 \frac{\alpha_{j}(t)}{\beta_{j}(t)} \times \frac{s_{1} e^{s_{1} x+\int s_{1}^{2} \alpha_{j}(t) d t}+s_{2} e^{s_{2} x+\int s_{2}^{2} \alpha_{j}(t) d t}}{1-e^{s_{1} x+\int s_{1}^{2} \alpha_{j}(t) d t}-e^{s_{2} x+\int s_{2}^{2} \alpha_{j}(t) d t}}, \quad j=1,2,3, \ldots, n .
$$

For three-singular-kink solutions, we use

$$
a_{j}(x, t)=1-e^{s_{1} x+\int s_{1}^{2} \alpha_{j}(t) d t}-e^{s_{2} x+\int s_{2}^{2} \alpha_{j}(t) d t}-e^{s_{3} x+\int s_{3}^{2} \alpha_{j}(t) d t}
$$

Proceeding as before, the three-singular-kink solutions for Eq. (2) are given by

$$
w_{j}(x, t)=-2 \frac{\alpha_{j}(t)}{\beta_{j}(t)} \times \frac{s_{1} e^{s_{1} x+\int s_{1}^{2} \alpha_{j}(t) d t}+s_{2} e^{s_{2} x+\int s_{2}^{2} \alpha_{j}(t) d t}+s_{3} e^{s_{3} x+\int s_{3}^{2} \alpha_{j}(t) d t}}{1-e^{s_{1} x+\int s_{1}^{2} \alpha_{j}(t) d t}-e^{s_{2} x+\int s_{2}^{2} \alpha_{j}(t) d t}-e^{s_{3} x+\int s_{3}^{2} \alpha_{j}(t) d t}}, \quad j=1,2,3, \ldots, n .
$$

In general, we can set $N$-singular-kink solutions for Eq. (2) as

$$
w_{j}(x, t)=-2 \frac{\alpha_{j}(t)}{\beta_{j}(t)} \times \frac{\sum_{i=1}^{N} s_{i} e^{s_{i} x+\int s_{i}^{2} \alpha_{j}(t) d t}}{1-\sum_{i=1}^{N} e^{s_{i} x+\int s_{i}^{2} \alpha_{j}(t) d t}}, \quad j=1,2,3, \ldots, n
$$

\section{Stabilities and propagation characteristics of solitary waves}

In this section, we discuss the effect of non-homogeneities, namely, variable coefficients to the nc-BE. The dispersion relation will be used to give the characteristic line and velocity $v$ for every soliton. The soliton amplitude $a m p$ for $w_{j}(x, t), j=1,2,3, \ldots, n$, can be expressed as

$$
a m p=\left|2 s_{1} \frac{\alpha_{j}(t)}{\beta_{j}(t)}\right|
$$




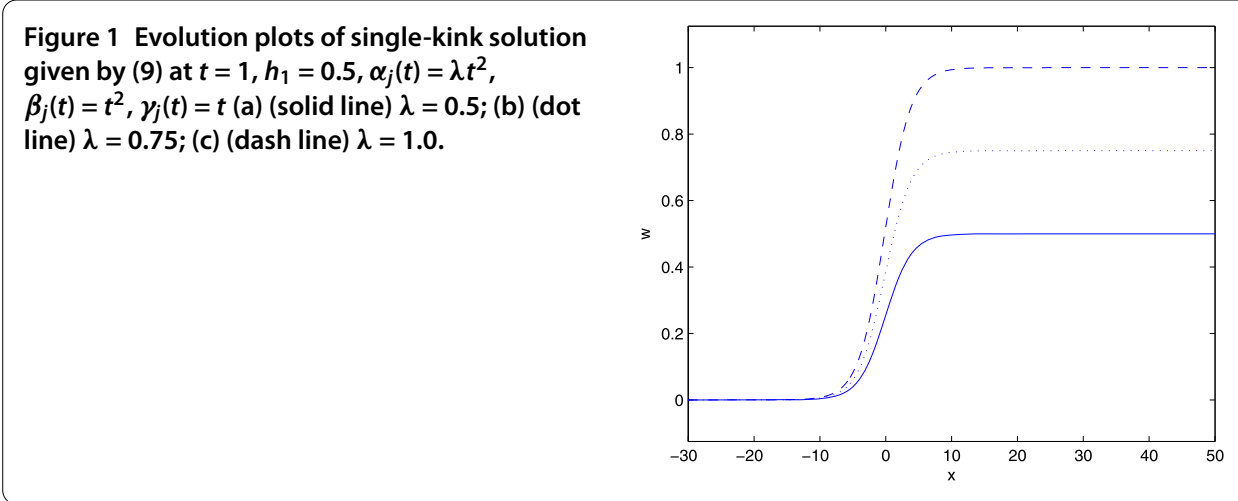

Figure 2 The profile figure for solution (12) with $s_{1}=0.5, s_{2}=0.75, \alpha_{j}(t)=\frac{8 t}{5 \Gamma(1.8)}$ and $\beta_{j}(t)=\frac{4 t}{5 \Gamma(1.8)}$.

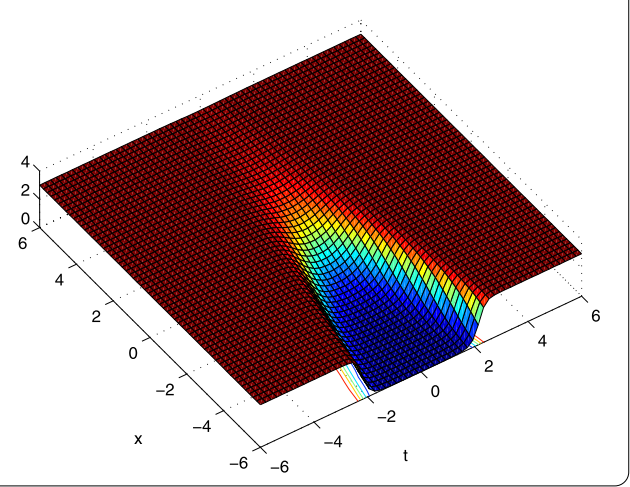

Using the characteristic-line method [31, 32], the characteristic wedge for each solitary wave for $w_{j}(x, t)$ is defined by

$$
x=-\int s_{i} \alpha_{j}(t) d t
$$

The velocity $v$ of each solitary wave for $w_{j}(x, t), j=1,2,3, \ldots, n$, is

$$
v_{x}=-s_{i} \alpha_{j}(t)
$$

The soliton amplitude amp depends on the variable coefficients $\alpha_{j}(t)$ and $\beta_{j}(t)$ but not on the variable coefficient $\gamma_{j}(t)$, see Figure 1 . The propagation velocity of the solitary wave Eq. depends only on the coefficient functions $\alpha_{j}(t)$. Moreover, we see that from (19), as the inequality $-s_{i} \alpha_{j}(t)>0$ holds, the soliton will move in the direction of positive $x$-axis.

In Figure 2, we choose $s_{1}=0.5, s_{2}=0.75, \alpha_{j}(t)=\frac{8 t}{5 \Gamma(1.8)}$ and $\beta_{j}(t)=\frac{4 t}{5 \Gamma(1.8)}$. Then the characteristic curve of Eq. (18) is given by

$$
x+\frac{4 s_{i}}{5 \Gamma(1.8)} t^{2}+\eta=0 .
$$

Then the soliton reveals the parabolic type propagation trajectory with unalterable amplitude but continuously changeable velocity. 
Figure 3 The profile figure for solution (12) with $s_{1}=0.5, s_{2}=0.75, \alpha_{j}(t)=\frac{7 \sin t}{10 \Gamma(1.4)}$, and $\beta_{j}(t)=\frac{7 \sin t}{20 \Gamma(1.4)}$.

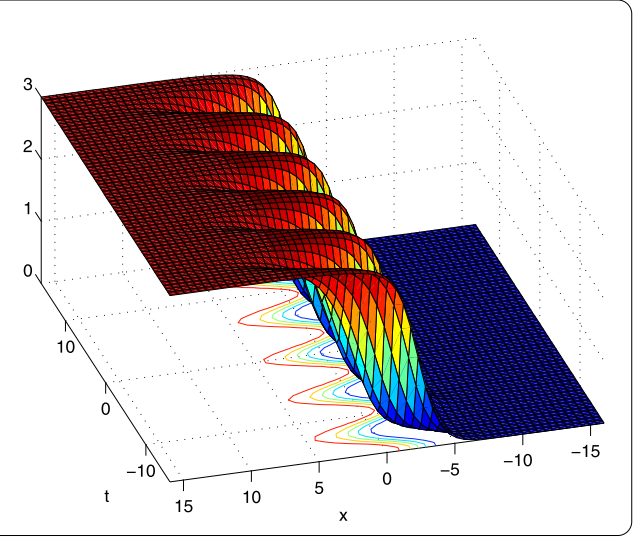

Figure 4 The propagation of two-kink solution (12) with $s_{1}=0.25, s_{2}=0.5, \alpha_{j}(t)=\frac{\sqrt{\pi}}{2}\left(-t^{2}+t-1\right)$ and $\beta_{j}(t)=\frac{\sqrt{\pi}}{2}\left(t^{2}-t+1\right)$.

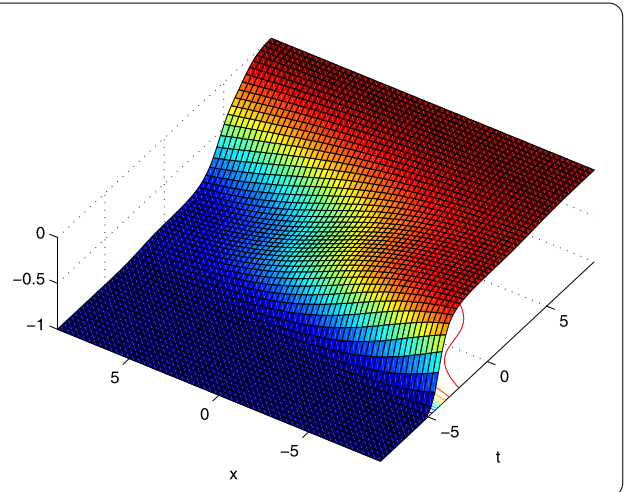

In Figure 3, we choose $s_{1}=0.5, s_{2}=0.75, \alpha_{j}(t)=\frac{7 \sin t}{10 \Gamma(1.4)}$ and $\beta_{j}(t)=\frac{7 \sin t}{20 \Gamma(1.4)}$. Then the characteristic curve of Eq. (18) is given by

$$
x-\frac{7 h_{i}}{10 \Gamma(1.4)} \cos t+\eta=0 .
$$

We see from Figure 3 that the propagation trajectory of the soliton presents the periodicity oscillation.

In Figures 4 and 5, we use Eq. (12) to discuss the interaction between two solitonic waves in a nonhomogeneous situation. In Figure 4 the interaction is called the overtaking coalescence. In this figure, we choose $s_{1}=0.25, s_{2}=0.5, \alpha_{j}(t)=\frac{\sqrt{\pi}}{2}\left(-t^{2}+t-1\right)$ and $\beta_{j}(t)=\frac{\sqrt{\pi}}{2}\left(t^{2}-t+1\right)$. The two fronts with the same propagation direction in $x$-axis coalesce into one large front in their interaction region of the $(x, t)$-plane, of which the amplitude amounts to two initial amplitudes. The front with faster velocity overtakes the slow-velocity one. In Figure 5, we choose $s_{1}=-0.25, s_{2}=0.5, \alpha_{j}(t)=\frac{\sqrt{\pi}}{2}\left(-t^{2}+t-1\right)$ and $\beta_{j}(t)=\frac{\sqrt{\pi}}{2}\left(t^{2}-t+1\right)$. The interaction is called head-on collision between one left-going soliton and one right-going soliton. Moreover, the directions of the solitary are controlled by the sign of velocity. It is clear that the amplitude and velocity after the collision of each soliton are not changed since the phase shift $b_{12}=0$.

\section{Conclusions}

In this work, we obtain new $N$-kink solutions and $N$-singular-kink solutions for new couplings of the Burgers equations with time-dependent variable coefficients (nc-BE) by using the simplified Hirota method and Backlund transformations. The condition 
Figure 5 The propagation of two-kink solution (12) with $s_{1}=-0.25, s_{2}=0.5, \alpha_{j}(t)=\frac{\sqrt{\pi}}{2}\left(-t^{2}+t\right.$ $-1)$ and $\beta_{j}(t)=\frac{\sqrt{\pi}}{2}\left(t^{2}-t+1\right)$.

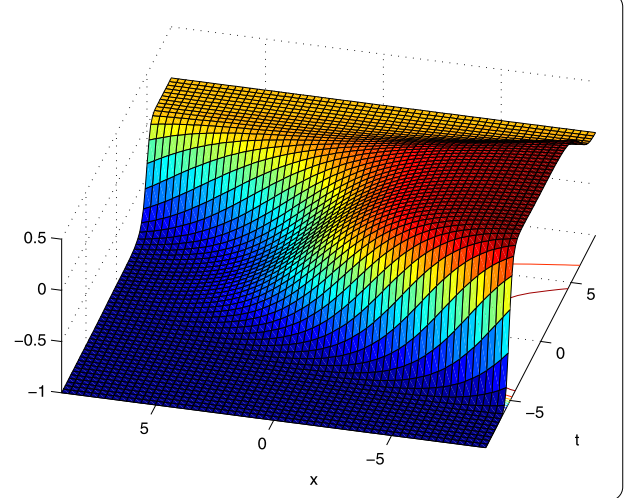

$\alpha_{j}(t)=b_{j} \beta_{j}(t)$ for Eq. (2) is sufficient to have multi-soliton solutions. We show the effect of time-dependent coefficients on amplitude and velocity of a single wave. We see that the amplitude depends on $\alpha_{j}(t)$ and $\beta_{j}(t)$, but the velocity of the wave depends only on $\alpha_{j}(t)$ and both of them are independent of $\gamma_{j}(t)$. Furthermore, the interaction behaviors and propagation characteristics of the solitons have been discussed. We see that the forms of the variable coefficients determine the appearances of the characteristic curve and correspond to distinct propagation trajectories.

Since the problem of bidirectional solitary waves has been reported in waves, in bubbly liquids [33, 34] and shallow-water waves [32], it is expected that the bidirectional solitonlike solutions to Eq. (2) are used to describe such interesting physical phenomena.

Regarding the complexity of the proposed problem, we highlight the main advantages of the proposed method:

1. The solution in the proposed method can be written in the exponential form, which generates multiple solutions, while other methods generate only single solution.

2. The proposed method shows the integrability of the modified equations, which is not possible in other methods.

3. In the proposed method, we use auxiliary functions to identify the type of the obtained solution, which is not possible in other methods.

4. The computational cost for the proposed method is cheaper compared with other methods.

Finally, most of the solitary wave methods give only single solution, either of type soliton, singular-soliton, kink, singular-kink, periodic or singular-periodic. Examples of these methods are the tanh expansion method, the sine-cosine method, the rational trigonometric function method, the tanh-sech function method, the $\left(G^{\prime} / G\right)$-expansion method, Jacobi elliptic function method and others [35-41]. The obtained solutions are always single. But, for the bilinear method, it gives multiple solutions at once.

Acknowledgements

The author would like to express his sincere gratitude to the editor and the reviewers for their valuable comments.

Competing interests

The author declares that they have no competing interests.

Publisher's Note

Springer Nature remains neutral with regard to jurisdictional claims in published maps and institutional affiliations. 


\section{References}

1. Nee, J, Duan, J: Limit set of trajectories of the coupled viscous Burger's equations. Appl. Math. Lett. 11(1), 57-61 (1998)

2. Abazari, R, Abazari, R: Numerical study of some coupled PDEs by using differential transformation method. Int. J. Math. Comput. Phys. Electr. Comput. Eng. 4(6), 641-648 (2010)

3. Esipov, SE: Coupled Burgers equations: a model of polydispersive sedimentation. Phys. Rev. E 52, 3711-3718 (1995)

4. Abdoua, MA, Solimanb, AA: Variational iteration method for solving Burger's and coupled Burger's equations. J. Comput. Appl. Math. 181, 245-251 (2005)

5. Dehghan, M, Hamidi, A, Shakourifar, M: The solution of coupled Burger's equations using Adomian-Pade technique. Appl. Math. Comput. 189(2), 1034-1047 (2007)

6. Abdul-Zahra, KA: Extended exponential function method in rational form for exact solution of coupled Burgers equation. J. Basrah Res. Sci. 38(1), 72-78 (2012)

7. Alomari, AK, Noorani, MSM, Nazar, R: The homotopy analysis method for the exact solutions of the $K(2,2)$, Burgers and coupled Burgers equations. Appl. Math. Sci. 2(40), 1963-1977 (2008)

8. Soliman, AA: The modified extended direct algebraic method for solving nonlinear partial differential equations. Int. J. Nonlinear Sci. 6(2), 136-144 (2008)

9. Al-Saif, AJS, Abdul-Hussein, A: Generating exact solutions of two-dimensional coupled Burgers' equations by the first integral method. Res. J. Phys. Appl. Sci. 1(2), 29-33 (2012)

10. Kumar, A, Arora, R: Solutions of the coupled system of Burgers' equations and coupled Klein-Gordon equation by RDT method. Int. J. Adv. Appl. Math. Mech. 1(2), 133-145 (2013)

11. Zuo, J-M: The Hirota bilinear method for the coupled Burgers equation and the high order Boussinesq-Burgers equation. Chin. Phys. B 20(1), 010205 (2011)

12. Hirota, R: Direct methods in soliton theory. In: Bullough, RK, Caudrey, PJ (eds.) Solitons. Springer, Berlin (1980)

13. Hirota, R: Exact N-soliton solutions of a nonlinear wave equation. J. Math. Phys. 14(7), 805-809 (1973)

14. Hirota, R: Exact solutions of the Korteweg-de Vries equation for multiple collisions of solitons. Phys. Rev. Lett. 27, 1192-1194 (1971)

15. Hirota, R: Exact N-soliton solutions of a nonlinear wave equation. J. Math. Phys. 14, 805-809 (1973)

16. Hirota, R: Exact solution of the modified Korteweg-de Vries equation for multiple collisions of solitons. J. Phys. Soc Jpn. 33, 1456-1458 (1972)

17. Awawdeh, F, Jaradat, HM, Al-Shara', S: Applications of a simplified bilinear method to ion-acoustic solitary waves in plasma. Eur. Phys. J. D 66(40), 1-8 (2012)

18. Alquran, M, Jaradat, HM, Al-Shara', S, Awawdeh, F: A new simplified bilinear method for the N-soliton solutions for a generalized FmKdV equation with time-dependent variable coefficients. Int. J. Nonlinear Sci. Numer. Simul. 16(6), 259-269 (2015)

19. Jaradat, HM, Awawdeh, F, Al-Shara', S, Alquran, M, Momani, S: Controllable dynamical behaviors and the analysis of fractal Burgers hierarchy with the full effects of inhomogeneities of media. Rom. J. Phys. 60, 324-343 (2015)

20. Awawdeh, F, Al-Shara', S, Jaradat, HM, Alomari, AK, Alshorman, R: Symbolic computation on soliton solutions for variable coefficient quantum Zakharov-Kuznetsov equation in magnetized dense plasmas. Int. J. Nonlinear Sci. Numer. Simul. 15(1), 35-45 (2014)

21. Alsayyed, $\mathrm{O}$, Jaradat, HM, Jaradat, MMM, Mustafa, Z, Shatat, F: Multi-soliton solutions of the BBM equation arisen in shallow water. J. Nonlinear Sci. Appl. 9(4), 1807-1814 (2016)

22. Wazwaz, AM: Multiple soliton solutions for the $(2+1)$-dimensional asymmetric Nizhnik Novikov Veselov equation. Nonlinear Anal. 72, 1314-1318 (2010)

23. Wazwaz, AM: Completely integrable coupled KdV and coupled KP systems. Commun. Nonlinear Sci. Numer. Simul. 15, 2828-2835 (2010)

24. Wazwaz, AM: Multiple-soliton solutions for the Boussinesq equation. Appl. Math. Comput. 192, 479-486 (2007)

25. Hereman, W, Zhuang, W: A macsyma program for the Hirota method, 13th World Congress. Comput. Appl. Math. 2 842-863 (1991)

26. Hietarinta, J: A search for bilinear equations passing Hirota's three-soliton condition. II. mKdV-type bilinear equations. J. Math. Phys. 28, 2094-2101 (1987)

27. Alquran, M: Solitons and periodic solutions to nonlinear partial differential equations by the sine-cosine method. Appl. Math. Inf. Sci. 6(1), 85-88 (2012)

28. Jaradat, HM, Al-Shara', S, Awawdeh, F, Alquran, M: Variable coefficient equations of the Kadomtsev-Petviashvili hierarchy: multiple soliton solutions and singular multiple soliton solutions. Phys. Scr. 85, 035001 (2012)

29. Jaradat, HM: New solitary wave and multiple soliton solutions for the time-space fractional Boussinesq equation. Ital. J. Pure Appl. Math. 36, 367-376 (2016)

30. Jaradat, HM: Dynamic behavior of traveling wave solutions for a class for the time-space coupled fractional kdV system with time-dependent coefficients. Ital. J. Pure Appl. Math. 36, 945-958 (2016)

31. Veksler, A, Zarmi, Y: Wave interactions and the analysis of the perturbed Burgers equation. Physica D 211, 57-73 (2005)

32. Yu, X, Gao, YT, Sun, ZY, Liu, Y: N-soliton solutions, Bäcklund transformation and Lax pair for a generalized variable-coefficient fifth-order Korteweg-de Vries equation. Phys. Scr. 81, 045402 (2010)

33. Miksis, MJ, Tinq, L: Effective equations for multiphase flows-waves in bubbly liquid. Adv. Appl. Mech. 28, 141-260 (1991)

34. Miksis, MJ, Tinq, L: Wave propagation in a bubbly liquid at small volume fraction. Chem. Eng. Commun. 118, 59-73 (1992)

35. Krishnan, EV: Remarks on a system of coupled nonlinear wave equations. J. Math. Phys. 31, 1155-1156 (1990)

36. Alquran, M, Qawasmeh, A: Soliton solutions of shallow water wave equations by means of ( $\left.G^{\prime} / G\right)$-expansion method. J. Appl. Anal. Comput. 4(3), 221-229 (2014)

37. Qawasmeh, A, Alquran, M: Reliable study of some new fifth-order nonlinear equations by means of $\left(G^{\prime} / G\right)$-expansion method and rational sine-cosine method. Appl. Math. Sci. 8(120), 5985-5994 (2014)

38. Shukri, S, Al-Khaled, K: The extended tanh method for solving systems of nonlinear wave equations. Appl. Math. Comput. 217(5), 1997-2006 (2010) 
39. Qawasmeh, A, Alquran, M: Soliton and periodic solutions for $(2+1)$-dimensional dispersive long water-wave system. Appl. Math. Sci. 8(50), 2455-2463 (2014)

40. Alquran, M, Ali, M, Al-Khaled, K: Solitary wave solutions to shallow water waves arising in fluid dynamics. Nonlinear Stud. 19(4), 555-562 (2012)

41. Alquran, M, Qawasmeh, A: Classifications of solutions to some generalized nonlinear evolution equations and systems by the sine-cosine method. Nonlinear Stud. 20(2), 263-272 (2013)

Submit your manuscript to a SpringerOpen ${ }^{\circ}$ journal and benefit from:

- Convenient online submission

- Rigorous peer review

- Open access: articles freely available online

- High visibility within the field

- Retaining the copyright to your article

Submit your next manuscript at $\boldsymbol{~ s p r i n g e r o p e n . c o m ~}$ 